\title{
Herbal Medicine Treatment of Refractory Epilepsy in Tuberous Sclerosis Complex : A Case Report
}

\author{
Kwanghyun Son, Jinsoo Lee, Moonju Kim \\ Department of Pediatric Neurology of Korean Medicine, Dong Seo Yung Hap (DSYH) Hospital, Seoul, Korea
}

\begin{abstract}
Infants with tuberous sclerosis complex (TSC) have a higher chance of experiencing seizures before the age of 1 year; in particular, they commonly accompany infantile spasms. In cases where infantile spasms resulting from TSC are drug-resistant, more severe neuro-developmental and cognitive impairments occur. This particular case dealt with an infant with TSC who continued to experience partial seizures and infantile spasms despite using two different kinds of antiepileptic drugs (AEDs). His spasms ceased on the seventh day of taking modified Yukmijihwang-tang (YMJ), at which point he stopped the use of all AEDs. He became seizure-free after a month of the treatment and modified hypsarrythmia was found to have been resolved in the electroencephalogram test. Until now, the infant has been taking YMJ for 16 months and is maintaining the seizure-free state without side effects. Moreover, his developmental status is continually improving, with a significant progress in language and cognitive-adaptive abilities. Such results suggest that YMJ can serve as an alternative treatment option for refractory epilepsy.
\end{abstract}

$\overline{\text { Key Words }}$ : tuberous sclerosis complex, infantile spams, refractory epilepsy, herbal medicine, developmental delay

\section{Introduction}

Tuberous sclerosis complex (TSC) is one of the most common types of neurocutaneous syndrome. Its major features include tumor-like lesions called hamartomas showing up in brains, hearts, kidneys or on skin. ${ }^{1)}$ The most common neurologic disorder that occurs in patients with TSC is seizure. Approximately $80-90 \%$ of TSC patients undergo seizures; in most cases, the onset of the first seizure occurs before the age of one year and they often accompany infantile spasms (IS). Early onset seizures and drug-resistant seizures like IS are known to leave more severe neuro-developmental and cognitive impairments. ${ }^{2}$

Yukmijihwang-tang is traditionally widely used as a prescription for mental and physical development delay in East Asia. In preclinical studies, it was found to increase neurogenesis and spatial memory functions and have neuro-protective effects. ${ }^{3-4)}$ Cheonma and Jogudeung, two other herbal ingredients, have anticonvulsant effects such that they are now receiving attention as less toxic alternatives of standard antiepileptic drugs (AEDs). ${ }^{5-6)}$ At Dong Seo Yung Hap (DSYH) hospital, by the process of the distinct diagnostic method in Korean traditional medicine, modified Yukmijihwang-tang (YMJ) Cheonma and Jogudeung added to Yukmijihwang-tang - is used as one of the treatments for epilepsy.

This case report was written to detail on the YMJ treatment on an infant with TSC and intractable

\footnotetext{
- Received : 29 May 2015

- Revised : 24 June 2015

- Accepted : 24 June 2015

- Correspondence to : Moonju Kim

Department of Pediatric Neurology of Korean Medicine, Dong Seo Yung Hap (DSYH) Hospital, Seoul, Korea

Tel : +82-2-2649-1140, Fax : +82-2-717-8363, E-mail : moonju-kim@hanmail.net
} 
epilepsy that resulted in a significantly positive seizure control and developmental improvement.

\section{Case}

Born a boy after a gestational period of 40 weeks by normal spontaneous vaginal delivery weighing $2.85 \mathrm{~kg}$, the subject was diagnosed with cardiac rhabdomyoma before birth and experienced his first seizure at his birth. The electroencephalogram (EEG) test detected nearly continuous epileptiform discharge in the right central area of his brain and diagnosed the subject with partial seizure two days after his birth. Accordingly, he began to take levetiracetam. The MRI test detected various sizes of tubers in the cortex and subependymal area, with the biggest lesion in his right frontal lobe at $2.5 \mathrm{~cm}$ (Figure 1). More than three hypomelanotic macules were observed throughout his body, and a genetic analysis showed deletion frameshift mutation in TSC2 gene which definitively confirmed the diagnosis of tuberous sclerosis. All of these tests and medications are conducted at the Department of Pediatrics, A Medical Center, University of U College of Medicine, Seoul.

A month after birth, he experienced seizures that were indicative of epileptic spasms as well as partial seizure. In the follow-up EEG, modified hypsarrythmia was confirmed and vigabatrin was added to his prescription with the IS diagnosis. However, epileptic spasms continued and developmental regression signs such as decreased eye-contact, cooing and smiling began to show.

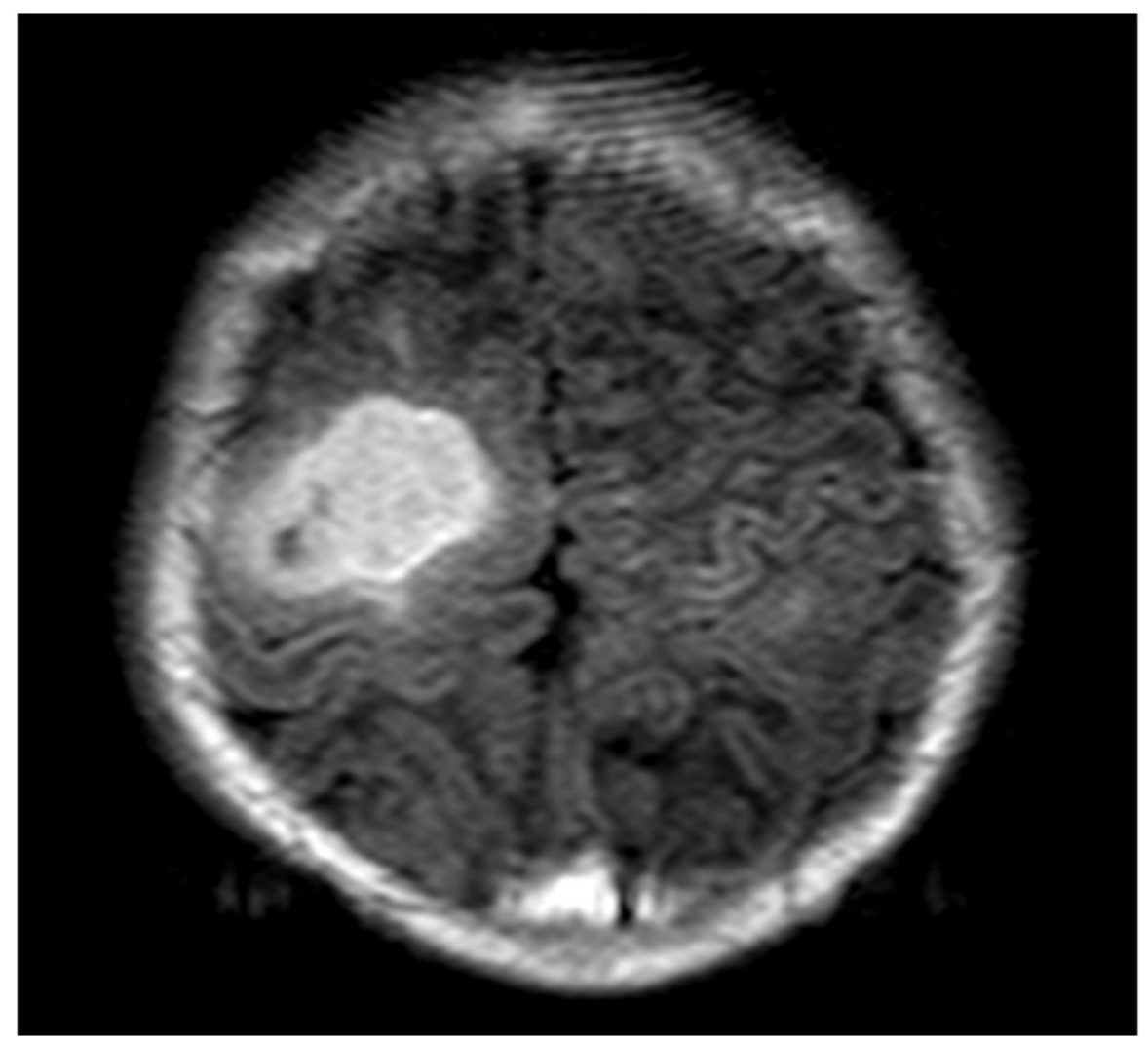

Fig. 1. Axial T1-weighted MRI shows an abnormal lesion on the right frontal cortex. 
At 2.5 months after birth (January 31th, 2014) when the subject first visited DSYH hospital he was taking $50 \mathrm{mg}$ of levetiracetam twice daily and 500 $\mathrm{mg}$ of vigabatrin twice a day. The subject was experiencing around 6-7 clusters with approximately 10 spasms per cluster, with a total of 60-70 epileptic spasms per day, and displaying developmental regression and delay. He showed levels of under one-month old in all five areas of the Korean Infant and Child Developmental Test (KICDT), which was developed to evaluate the development status of infant and child in terms of developmental age (DA) and developmental quotient (DQ). ${ }^{7)}$ He began to take YMJ and stopped using vigabatrin provisionally.

YMJ's daily dosage consists of the following: $4 \mathrm{~g}$ of Rehmanniae Radix (地黄), 3g of Dioscorae Radix (山藥), $3 \mathrm{~g}$ of Corni Fructus (山茱莫), $2 \mathrm{~g}$ of Alimatis Rhizoma (澤潟), $2 \mathrm{~g}$ of Moutan Cortex Radicis (牧丹 皮), $2 \mathrm{~g}$ of Hoelen (获苓), $1.5 \mathrm{~g}$ of Gastrodia elata (天 麻), and $1.5 \mathrm{~g}$ of Uncaria sinensis (釣鉤藤). These herbal medicinal ingredients were boiled in approximately $100 \mathrm{cc}$ of water at $102-103{ }^{\circ} \mathrm{C}$ for approximately 2 hours such that there was about 30 cc of liquid left. The solution was packaged by 10 cc and the subject was ordered to take it 3 times a day, 30 minutes after each meal.

Despite the discontinuation of vigabatrin usage, seizure frequency began to decrease with the use of YMJ; at one week of the YMJ treatment, the subject achieved cessation of spasms (Figure 2). Furthermore, when his movements increased and his eye-contact and neck-control began to improve, the infant's guardians decided to also stop the use of levetiracetam provisionally. Two weeks after withdrawing from levetiracetam the subject showed approximately 3-5 partial seizures per day over a week, each one lasting around 30 seconds. However, he continued to show positive developmental improvement with eye-tracking, responding to sound or his name and thus his progress was observed without using AEDs.

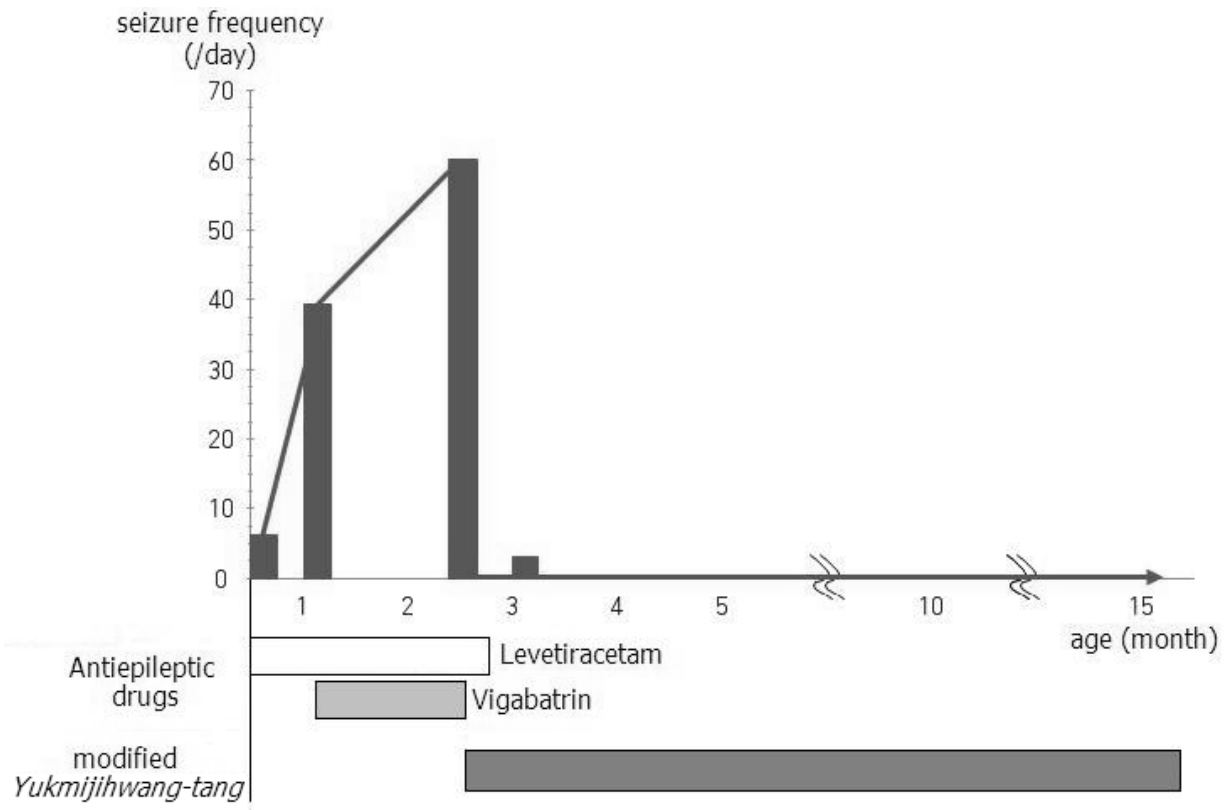

Fig. 2. Clinical course of the subject treated with modified Yukmijihwang-tang 
Table 1. Development Outcomes Assesed by Using Korean Infant and Child Developmental Test during the Clinical Course

\begin{tabular}{cccccc}
\hline \multirow{2}{*}{ Age } & \multicolumn{5}{c}{ Domain } \\
\cline { 2 - 6 } & Gross motor & Fine motor & Personal-social & Language & Cognitive-adaptive \\
\hline 2.5 months & 1 & 0 & 1 & 1 & 1 \\
4 months & 4 & 3 & 3 & 4 & 4 \\
8 months & 5 & 4 & 6 & 7 & 6 \\
14 months & 7 & 5 & 8 & 11 & 9 \\
\hline
\end{tabular}

Data are presented as developmental age (months) at each chronological age.

He reclaimed the seizure-free state after this period.

At 4 months old (1.5 month of YMJ treatment), when the child underwent the follow-up EEG at the general hospital at which he was first diagnosed with TSC, the test rendered resolution of modified hypsarrythmia. Moreover, the epilepticform discharge that was consistently present at the right central area of his brain was rarely observed this time.

At 5 months old, the child began rehabilitation treatment because the results of the KICDT test performed at DSYH hospital showed impaired fine motor skills of his left arm and hand. At 8 months old, he was diagnosed with left hemiplegia at the general hospital, which was assumed to be caused by TSC but by 14 months, he showed progress in his motor, language and cognitive-adaptive abilities, comparatively more in the latter two than the first (Table 1).

Last follow-up was conducted on May 15th, 2015 (18 months old). Until that time, the infant was continuing the YMJ treatment and rehabilitation for hemiplegia. He has not experienced side effects or adverse events during the past 15 months of treatment, and the trimonthly tests conducted on aspartate transaminase (AST), alanine transaminase (ALT), alkaline phospatase (ALP), gamma glutamyl tranapeptidase (GGT), total protein, albumin, total bilirubin, blood urea nitrogen (BUN), and creatinine levels all rendered results in the normal range.

\section{Discussion}

Infants with TSC experiencing IS are reported to show approximately $85-100 \%$ probability of mental retardation. ${ }^{8-10)}$ In particular, patients with TSC2 gene mutation experience more tubers, earlier seizure onset, and have higher probability of IS and intractable seizures - which could all lead to more profound intellectual disability - than those with TSC1 gene mutation. ${ }^{11)}$ In general, infants with TSC displaying IS are typically treated first with vigabatrin $^{12)}$, which shows approximately 50-90\% clinical response rate. ${ }^{13)}$

The subject in this study was found to have TSC2 gene mutation, began experiencing neonate seizure and progressed to IS, and was deemed to have a type of refractory epilepsy that does not respond to vigabatrin and levetiracetam. The fact that he achieved the seizure-free state without side effects or that he became "normal" by the EEG test after discontinuing the use of AEDs and only taking YMJ shows that YMJ has seizure control effects.

The subject also showed developmental progress only with the use of YMJ; this progress was far more significant in language and cognitive-adaptive abilities. Such result is likely due to the patient having a greater motor ability impairment and/or YMJ having more influence in the areas involving intellectual abilities rather than motor abilities.

Intellectual impairments that show up in TSC patients are generally attributed to the underlying pathology of TSC itself or to seizure. Most research 
up to this point has been focused on seizure control; however, the proportion of those whose cognitive impairment is due to seizure is reported to be below $50 \%{ }^{2}{ }^{2}$ Hence, research on mammalian target of rapamycin (mTOR) inhibitors that directly act on TSC's pathophysiological mechanisms are rendering some more productive results, though with limitations. ${ }^{14)}$ Considering that developmental regression and delay were observed in this study's subject while IS was in progress, and that his developmental improvement began after he became seizure-free with the YMJ treatment, seizure control seems to have at least a limited amount of influence on development outcomes. However, seeing as Yukmijihwang-tang, the largest part of YMJ's composition, has traditionally been used as a treatment for infant developmental impairment rather than for epilepsy, YMJ may have been more affective in developmental improvement from directly affecting the underlying pathology of TSC. More clinical and preclinical studies on such matters are needed in the near future.

\section{References}

1. Curatolo P, Verdecchia M, Bombardieri R. Tuberous sclerosis complex: a review of neurological aspects. European Journal of Paediatric Neurology. 2002;6:12-23.

2. O'Callaghan FJ, Harris T, Joinson C, Bolton P, Noakes M, Presdee D, et al. The relation of infantile spasms, tubers, and intelligence in tuberous sclerosis complex. Arch Dis Child. 2004;89:530-533.

3. Shin HT, Chung SH, Lee JS, Kim SS, Shin HD, Jang $\mathrm{MH}$, et al. Protective effect of shenqi-wan against $\mathrm{H} 2 \mathrm{O} 2$-induced apoptosis in hippocampal neuronal cells. The American Journal of Chinese Medicine. 2003;31:675-686.

4. Lee KS, Lim BV, Chang HK, Yang HY, Bahn GH, Paik EK, et al. Liuweidihuang-tang improves spatial memory function and increases neurogenesis in the dentate gyrus in rats. Fitoterapia. 2005;76:514-519.

5. Ojemann LM, Nelson WL, Shin DS, Rowe AO, Buchanan RA. Tian ma, an ancient Chinese herb, offers new options for the treatment of epilepsy and other conditions. Epilepsy \& Behavior. 2006;8(2):376-383.

6. Liu CH, Lin YW, Tang NY, Liu HJ, Hsieh CL. Neuroprotective effect of Uncaria rhynchophylla in kainic acid-induced epileptic seizures by modulating hippocampal mossy fiber sprouting, neuron survival, astrocyte proliferation, and S100B expression. Evid Based Complement Alternat Med. 2012;2012:194790.

7. Choi HW, Jeong MH, Lee EH, Jeong SJ, Yum MS, Ko TS. Korean infant and child developmental test (KICDT) in children with epilepsy. J Korean Child Neurol Soc. 2010; 18:40-48.

8. Fukushima K, Inoue Y, Fujiwara T, Yagi K. Long-term course of West syndrome associated with tuberous sclerosis. Epilepsia. 1998;39:50 -54 .

9. Husain AM, Foley CM, Legido A, Chandler DA, Miles DK, Grover WD. West syndrome in tuberous sclerosis complex. Pediatr Neurol. 2000;23:233-235.

10. Riikonen R, Simell O. Tuberous sclerosis and infantile spasms. Dev Med child Neurol. 1990; 32:203-209.

11. Bolton PF, Clifford C, Tye C, Maclean C, Humphrey A, le Marechal K, et al. Intellectual abilities in tuberous sclerosis complex: risk factors and correlates from the Tuberous Sclerosis 2000 Study. Psycological Medicine. 2015;1:1-11.

12. Pellock JM, Hrachovy R, Shinnar S, Baram TZ, Bettis D, Dlugos DJ, et al. Infantile spasms: A U.S. consensus report. Epilepsia. 2010;51:2175 -2189 .

13. Mackay MT, Weiss SK, ms-Webber T. Ashwal S, Stephens D, Ballaban-Gill K, et al. Practice 
parameter: medical treatment of infantile spasms: report of the American academy of neurology and the child neurology society. Neurology. 2004;62:1668-1681.

14. Cappellano A, Senerchia A, Adolfo F, Paiva P,
Pinho R, Covic A, et al. Successful everolimus therapy for SEGA in pediatric patients with tuberous sclerosis complex. Child's Nervous System. 2013;29:2301-2305. 